\title{
CT Appearance Following Partial Nephrectomy for Renal Cell Carcinoma Using Tissue Adhesive Bioglue"
}

\author{
Simona Croituro, Yair Braz, Boaz Moskovitz, Ofer Nativ, Sarel Halachmi \\ Departments of Radiology and Urology, Bnai Zion Medical Center, Faculty of Medicine, Technion, \\ Israeli Institute of Technology, Haifa, Israel \\ Email: yair_braz@hotmail.com
}

Received December 27, 2012; revised January 31, 2013; accepted February 7, 2013

Copyright (C) 2013 Simona Croituro et al. This is an open access article distributed under the Creative Commons Attribution License, which permits unrestricted use, distribution, and reproduction in any medium, provided the original work is properly cited.

\begin{abstract}
Background: The use of biological sealants has greatly increased during nephron sparing surgery. In many cases the bulk of the material was erroneously mistaken for tumor recurrence. Objective: To describe the characteristic appearance of biological adhesive material used for tumor bed closure on computerized tomography (CT) following nephrone sparing surgery (NSS) for renal cell carcinoma, in order to differentiate between typical features of the adhesive material and local tumor recurrence. Design, Setting and Participants: We retrospectively reviewed follow-up CT scans of 120 patients who underwent NSS for T1N0M0 RCC. In all cases tumor bed was closed during surgery with biological tissue adhesive (BioGlue). Results and Limitations: During 1994-2009, 120 patients with a single T1 renal cell carcinoma lesion, underwent NSS with closure of tumor bed with bio adhesive material. There were 66 males and $47 \mathrm{fe}-$ males with mean age of 58.7 years (median: 58 years, range: 28 - 85 years). Mean follow-up time was $45 \pm 34$ months (median 42, range 12 - 168). During follow-up, 3 patients had local recurrence at the site of previous enucleated lesion. In the first post-operative CT scan the BG appeared as a heterogeneous mass with sharp edges measuring $20-70 \mathrm{HU}$ with no attenuation following the injection of contrast material. In subsequent follow-up scans the BG in most patients remained stable in size; in few patients slight reduction in size was observed probably due to the resolution of post-operative hematoma. Tumor recurrence that was documented in 3 patients was seen as a heterogeneous mass with attenuation of more than $20 \mathrm{HU}$ following the injection of contrast material. In sequential CT's the mass was increasing in size. Conclusions: BG appears as a non-enhancing stable mass in sequential CT's following NSS, hence could be differentiated from local tumor recurrence. The ability to differentiate between normal post-operative status and recurrence could be compromised in patients with decreased renal function in whom contrast material could not be used.
\end{abstract}

Keywords: CT; RCC; Tissue Adhesive Bioglue

\section{Introduction}

Nephron sparing surgery (NSS) is now considered as the preferable treatment option for most patients with organ confined renal cancer. Such approach provides excellent cancer control as compared with radical nephrectomy with the advantage of renal function preservation [1,2]. Closure of tumor bed and hemostasis can be achieved by either conventional surgical techniques such as suture ligature, cautery, and argon beam coagulation or by application of various types of hemostatic agents [3]. The main goals in NSS are cancer control and the preservation of renal function. Following tumor enucleation several surgical techniques are used. We have previously demonstrated that the use of BioGlue (BG) (Cryolife,

*All authors contributed equally to this manuscript.
Atlanta, Ga, USA) tissue adhesive for closure the parenchymal defect during NSS provides excellent hemostatatic control, prevents urine leak and increases the possibility to preserve maximal functioning renal tissue $[4,5]$. The BG mass in post-operative follow-up CT scans appears as a heterogeneous mass raising false positive suspicions for local tumor recurrence. Our aim of study was to describe the typical radiological appearance of $B G$ in sequential post-operative scans and to differentiate it from the tomographic features of local tumor recurrence.

\section{Materials and Methods}

Between 1994 and 2009, 229 consecutive patients underwent NSS for localized RCC among them in 113 tumor bed closure was done using $\mathrm{BG}$. We retrospectively 
collected data including age, gender, and symptoms at presentation, operative time, warm ischemia time, blood loss, surgical complications, tumor size, Fuhrman grade, TNM stage, margin status, disease relapse and patients' outcome. Preoperative evaluation in all patients included physical examination, laboratory analysis and chest, abdomen and pelvic CT scan without and with intra venous contrast.

\subsection{CT Protocol}

The studies were performed using a standard CT Urography protocol: slice thickness: $5 \mathrm{~mm}(128 \times 0.6 \mathrm{~mm})$, $120 \mathrm{kV}, 200 \mathrm{mAs}$, and pitch: 0.61. Contrast administration: $80 \mathrm{cc}$ Iomeron 350, injection rate: $3.5 \mathrm{ml} / \mathrm{sec}$, delay: $300 \mathrm{sec}$. All studies were read and review by an experienced uro-radiologist (S.C.), and 2 urologists (S.H., O.N.).

\subsection{Operative Technique}

The open surgical procedure for NSS included lateral retroperitoneal approach, removal of the perirenal fat that was sent for pathological examination, identification of the suspected renal mass and isolation of the renal pedicle. Before renal vessels clamping intra-venous manitol was given $(0.5 \mathrm{gr} / \mathrm{Kg}$ body weight) followed by surface hypothermia with ice slush for 20 minutes. The lesion was removed with a rim of minimal normal renal tissue. Samples from the tumor base were sent for frozen section analysis. Open blood vessels or collecting system were sutured using monocryle 4/0; argon beam coagulator was used to seal the exposed renal parenchyma, 5-10 $\mathrm{ml}$ of BG tissue adhesive were used to fill the tumor bed.

\subsection{Follow-Up}

Follow-up protocol included CT scan without and with intra venous contrast. In our practice we perform the $1^{\text {st }}$ post-operative $\mathrm{CT}$ at 3 months after surgery and then every 6 months during the first 2 years post-surgery, thereafter annually. In all patients we had preoperative CT scan and at least 2 post-operative scans.

\section{Results}

Between 1994-2009, 113 patients with a single T1 renal cell carcinoma lesion, underwent NSS with closure of tumor bed with BG. There were 66 males and 47 females with mean age of 58.7 years (median: 58 years, range: 28 85 years). Eleven patients had a single kidney. Mean follow-up time was $45 \pm 34$ months (median 42, range 12 168). During follow-up, 3 patients had local recurrence at the site of previous enucleated lesion.

In the first post-operative CT scan the BG appeared as a heterogeneous mass with sharp edges measuring 20 $70 \mathrm{HU}$ with no attenuation following the injection of contrast material. The BG mass could be engulfed by residual post-operative bleeding or hematoma. In later follow-up scan the BG in most patients remained stable in size; in few patients we observed slight reduction in size probably due to the resolution of post-operative hematoma.

In contrast tumor recurrence that was documented in 2 patients was seen as a heterogenous mass with attenuation of more than $20 \mathrm{HU}$ following the injection of contrast material. In sequential CT's the mass was increasing in size.

\section{Discussion}

The incidence of renal masses and renal cancer has persistently increased in recent years, mainly due to the use of non-invasive diagnostic imaging modalities for the evaluation of abdominal and musculoskeletal symptoms. Most of these incidentally detected tumors (70\%) are small asymptomatic and confined to the kidney [6]. In parallel there is increasing data in the literature that demonstrate association between postoperative renal function and non-cancer mortality mainly due to cardiovascular events. For these reasons Nephron sparing surgery (NSS) has become the treatment of choice for stage T1 localized renal cell carcinoma [7,8].

Several surgical methods exist for achieving hemostasis following tumor enucleation including: the traditional closure of the parenchymal defect with suture ligasure by application of topical absorbable hemostats such as oxidized regenerated cellulose, gelatin, collagen and liquid fibrin sealants have been employed to decrease intraoperative renal ischemic time, provide rapid hemostasis and improve visualization of the surgical field [5,9-12]. In our series we have used BG to fill the parenchymal defect. The BG is a surgical adhesive composed of purified bovine serum albumin (BSA) and glutaraldehyde. The two components are dispensed by a controlled delivery system comprising a double-chambered syringe. Once dispensed, the adhesive components are mixed within the applicator tip where the cross-linking begins. The glutaraldehyde molecules bond with the BSA molecules and, upon application to the tissue proteins at the repair site, create a flexible mechanical seal independent of the body's clotting mechanism. It begins to polymerize within 20 to 30 seconds and reaches its bonding strength within two minutes. Its main advantage is the easy application as the mixed product could be easily poured into the kidney even into non flat surface. The use of BG may eliminate of need for hemostatic sutures sparing more functional parenchyma. BG is widely used in NSS [13-15]. Nativ et al. [5] using pre and post-operative DMSA scan showed a marked difference in renal function loss when hemostatic sutures were used compare to 
BG. The post vs. pre-operation differential function reduction using BG was $-4.9 \%$ compare to $-13.4 \%$ with hemostatic sutures $(\mathrm{p}<0.06)$.

Oncological failure following NSS occurs and varies between $5 \%-15 \%$ in different series. Halachmi et al. reported about 5.6\% (13/229) recurrence rate following surgery. Hafez et al. [16] reported about $11.6 \%$ incidence of recurrence among 327 patients who underwent NSS for sporadic localized RCC. Van Poppel et al. [17] observed 3.94\% (3/76 patients) systemic progression rate, and Zigeuner et al. [18] reported about oncology failure in 17 of 114 patients (15\%) who underwent NSS. Postoperative oncological failure could be sub classified to: local recurrence at the surgical site, peri-renal recurrence, systemic recurrence, i.e. metastases, and the appearance of new renal lesion. Recurrence at the surgical site and peri-renal recurrence are challenging as these clinical conditions should be differentiated from normal postoperative changes. The use of BG further complicates the radiological diagnosis as the bulk of the adhesive material looks like a heterogeneous mass resembling recurrence of renal carcinoma. Recurrence at the surgical site and peri-renal recurrence should be recognized as soon as possible and treated surgically or by ablative energy allowing adequate cancer control.

CT scan of the abdomen is considered the gold standard imaging technique for post NSS surveillance [19]. CT findings after NSS are different from those shown after radical nephrectomy [20], however, there is little data in the literature concerning CT findings after renal tumor enucleation using BG to close the tumor bed. In our practice we see many patients diagnosed falsely with tumor recurrence as the BG balk is misinterpreted as viable tissue, As the majority of patients will have their CT done and analyzed in radiology clinic outside the hospital we noticed that sometimes radiologist define post-operative changes created by the BG false positively as tumor recurrence, we therefore decided to analyze and show the crucial difference between those two distinct states.

As 5 - $15 \mathrm{ml}$. of BG are usually used to fill the defect in the parenchyma it may appear as a new lesion on an unenhanced CT (Figure 1) or US. We noticed that in the first post-operative CT scan residual post-operative bleeding or hematoma may engulf the BG, these findings disappear in following examinations. The BG appears as a heterogeneous mass with sharp edges measuring 20 - 70 HU with no attenuation following the injection of contrast material (Figures 1 and 2). In later follow-up scans the $\mathrm{BG}$ in most patients remained stable in size, and in few of them we observed slight reduction in size probably due to the resolution of the post-operative hematoma. In contrast, tumor recurrence that was documented in 2 patients was seen as a heterogeneous mass with attenuation of more than $20 \mathrm{HU}$ (on average X HU) following the injection of contrast material (Figure 3). In sequential CT's one of the mass was also increasing in size.

With no exception in all patients the BG did not change its initial density (HU count) and it did not enhance nor in the vascular neither in the late excretion phases of the CT.

The differential diagnosis of renal masses following NSS is wide, Lee et al. [20] in a comprehensive paper described a wide variety of post-operative changes. Pai et al. [21] described the CT finding in the kidney following NSS using cellulose bolsters. Searching the medical literature we have not found a paper describing the changes following the use of $\mathrm{BG}$, as the use of this material rapidly growth we thought it will beneficial for the clinicians to be familiar with its radiological characteristic in order to differentiate between normal post-operative features and those of tumor recurrence.

\section{Conclusions}

There is a constant rise in NSS performed for localized RCC, despite the excellent surgical results local recur-

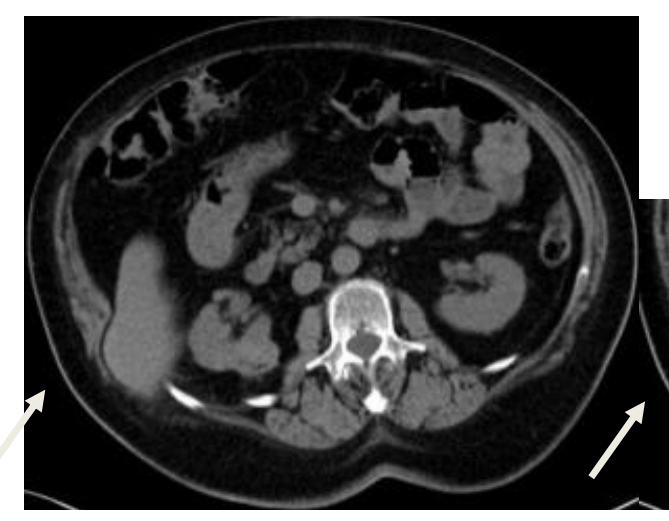

(a)

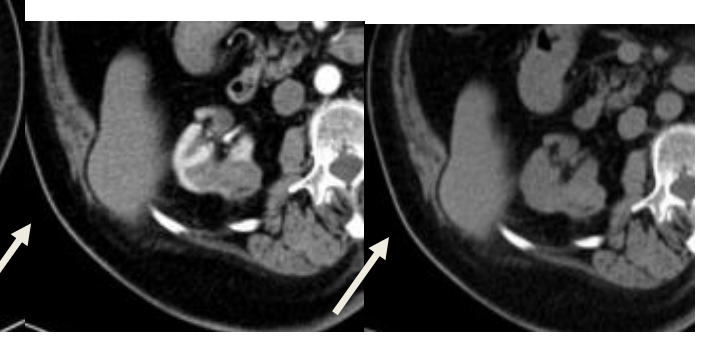

(b)

(c)

Figure 1. The BG appears as a heterogeneous mass with sharp edges measuring 20 - 70 HU with no attenuation following the injection of contrast material as seen in 1a before the injection. 1b following injection. 1c washout phase. 


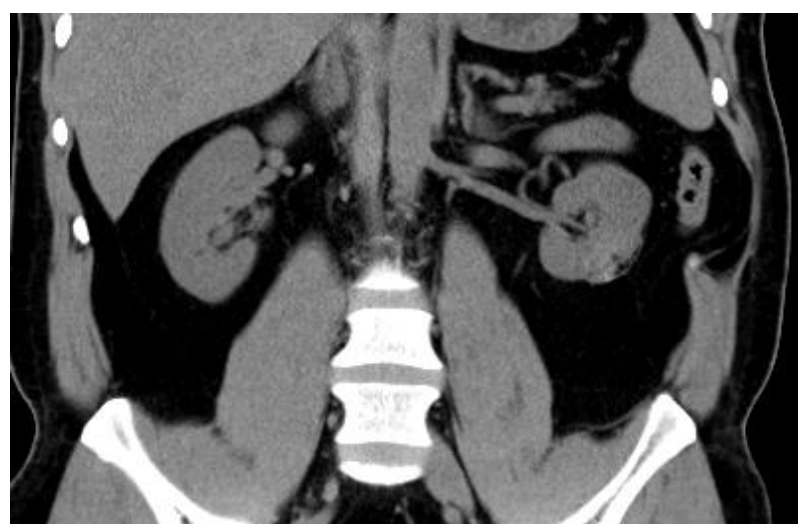

(a)

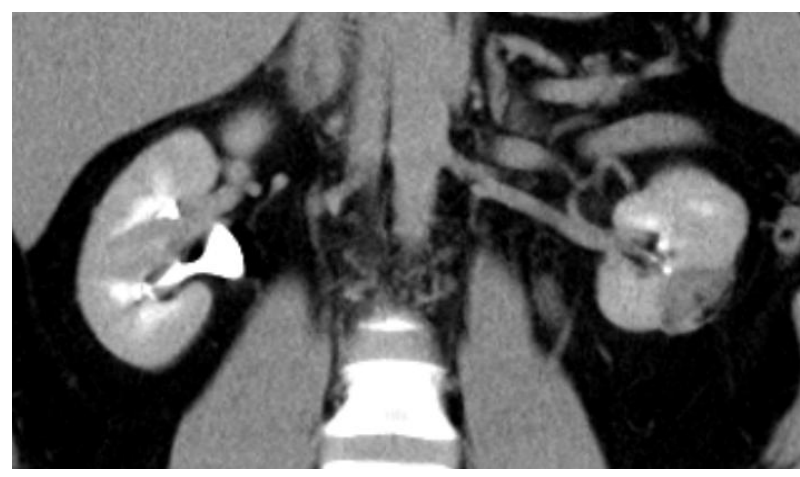

(b)

Figure 2. Another example of BG appears as a heterogeneous mass with no attenuation following the injection of contrast material. (a) Before the injection; (b) Following injection.

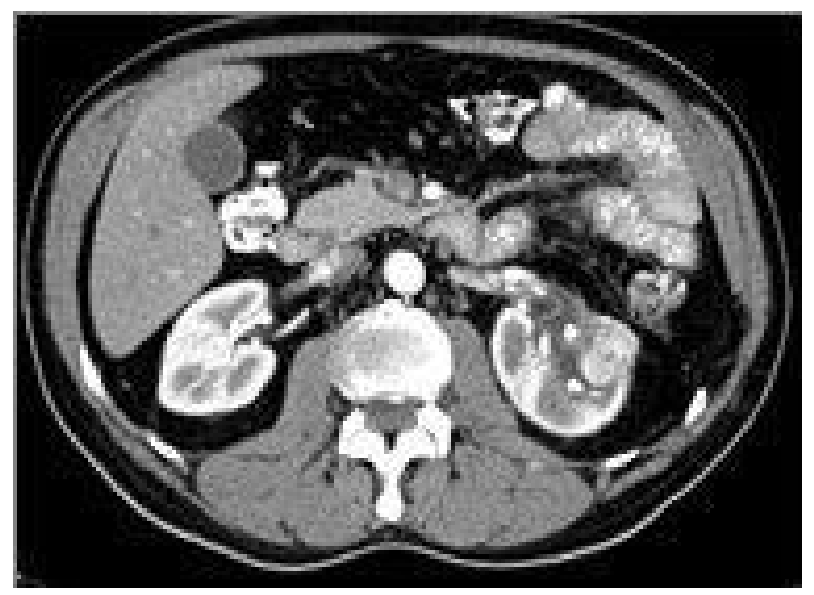

Figure 3. Tumor recurrence seen as a heterogeneous mass with attenuation of more than $20 \mathrm{HU}$.

rence at the enucleated site occurs. It is crucial to identify as soon as possible tumor recurrence as these patients can be salvaged by surgery or ablation. The best available modality to follow-up patients following NSS is sequential CT scans, hence it is essential to delineate the post-operative changes and due to the increased use in adhesive materials. We describe the post-operative findings following the use of BG in NSS demonstrating mainly the fact that it does not enhance in contrast to tumor recurrence.

\section{REFERENCES}

[1] F. Becker, S. Siemer, U. Humke, M. Hack, M. Ziegler and M. Stockle, "Elective Nephron Sparing Surgery Should Become Standard Treatment for Small Unilateral Renal Cell Carcinoma: Long-Term Survival Data of 216 Patients," European Urology, Vol. 49, No. 2, 2006, pp. 308-313. doi:10.1016/j.eururo.2005.10.020

[2] W. K. Lau, M. L. Blute, A. L. Weaver, V. E. Torres and H. Zincke, "Matched Comparison of Radical Nephrectomy vs Nephron-Sparing Surgery in Patients with Unilateral Renal Cell Carcinoma and a Normal Contralateral Kidney," Mayo Clinic Proceeding, Vol. 75, No. 12, 2000, pp. 1236-1242. doi:10.4065/75.12.1236

[3] F. Porpiglia, J. Renard, M. Billia, I. Morra, C. Terrone and R. M. Scarpa, "Biological Glues and Collagen Fleece for Hemostasis during Laparoscopic Partial Nephrectomy: Technique and Results of Prospective Study," Journal of Endourology, Vol. 21, No. 4, 2007, pp. 423-428. doi:10.1089/end.2006.0265

[4] G. Hidas, L. Lupinsky, A. Kastin, B. Moskovitz, D. Groshar and O. Nativ, "Functional Significance of Using Tissue Adhesive Substance in Nephron-Sparing Surgery: Assessment by Quantitative SPECT of $99 \mathrm{~m}$ Tc-Dimercaptosuccinic Acid Scintigraphy," European Urology, Vol. 52, No. 3, 2007, pp. 785-789.

doi:10.1016/j.eururo.2006.12.001

[5] O. Nativ, A. Levi, R. Farfara, S. Halachmi and B. Moskovitz, "Degree and Predictors of Functional Loss of the Operated Kidney following Nephron-Sparing Surgery: Assessment by Quantitative SPECT of $99 \mathrm{~m}$ Tc-Dimercaptosuccinic Acid Scintigraphy," Advances in Urology, Vol. 2011, 2011, Article ID: 961525.

[6] C. J. Kane, K. Mallin, J. Ritchey, M. R. Cooperberg and P. R. Carroll, "Renal Cell Cancer Stage Migration: Analysis of the National Cancer Data Base," Cancer, Vol. 113, No. 1, 2008, pp. 78-83. doi:10.1002/cncr.23518

[7] A. Antonelli, A. Cozzoli, M. Nicolai, D. Zani, T. Zanotelli, L. Perucchini, S. C. Cunico and C. Simeone, "Nephron-Sparing Surgery Versus Radical Nephrectomy in the Treatment of Intracapsular Renal Cell Carcinoma up to $7 \mathrm{~cm}$," European Urology, Vol. 53, No. 4, 2008, pp. 803-809. doi:10.1016/j.eururo.2007.11.007

[8] J. J. Patard, O. Shvarts, J. S. Lam, A. J. Pantuck, H. L. Kim, V. Ficarra, L. Cindolo, K. R. Han, T. A. De La, J. Tostain, W. Artibani, C. C. Abbou, B. Lobel, D. K. Chopin, R. A. Figlin, P. F. Mulders and A. S. Belldegrun, "Safety and Efficacy of Partial Nephrectomy for All T1 Tumors Based on an International Multicenter Experience," The Journal of Urology, Vol. 171, No. 6, 2004, pp. 2181-2185. doi:10.1097/01.ju.0000124846.37299.5e

[9] J. H. Kaouk, S. P. Hillyer, R. Autorino, G. P. Haber, T. Gao, F. Altunrende, R. Khanna, G. Spana, M. A. White, H. Laydner, W. Isac and R. J. Stein, "252 Robotic Partial 
Nephrectomies: Evolving Renorrhaphy Technique and Surgical Outcomes at a Single Institution," Urology, Vol. 78, No. 6, 2011, pp. 1338-1344.

[10] D. S. Park and W. K. Jang, "Secure Reconstruction Technique after Partial Nephrectomy Irrespective of Tumor Size and Location," International Brazilian Journal of Urology, Vol. 35, No. 4, 2009, pp. 416-426. doi:10.1590/S1677-55382009000400004

[11] J. Sammon, F. Petros, S. Sukumar, A. Bhandari, S. Kaul, M. Menon and C. Rogers, "Barbed Suture for Renorrhaphy during Robot-Assisted Partial Nephrectomy," Journal of Endourology, Vol. 25, No. 3, 2011, pp. 529533. doi:10.1089/end.2010.0455

[12] J. Simon, F. Finter, A. Ignatius, M. Meilinger and L. Durselen, "Maximum Tensile Force of Different Suture Techniques in Reconstruction of the Renal Remnant after Nephron-Sparing Surgery," Surgical Endoscopy, Vol. 25, No. 2, 2011, pp. 503-507. doi:10.1007/s00464-010-1201-0

[13] A. Celia, G. Zeccolini, G. Guazzoni, V. Pansadoro, V. Disanto, F. Porpiglia, C. Milani, C. Abbou, R. Gaston, G. Janetschek, N. A. Soomroo, P. Fornara, A. Breda, P. G. Schulam, R. J. De la, M. P. Laguna, J. Palou and G. Breda, "Laparoscopic Nephron Sparing Surgery: A MultiInstitutional European Survey of 592 Cases," Archives of Italian Urology and Andrology, Vol. 80, No. 3, 2008, pp. 85-91.

[14] H. Jung, J. P. Brusky and G. W. Chien, "BioGlue Presenting as Radiographic Emphysematous Pyelonephritis after Laparoscopic Partial Nephrectomy," Journal of Endourology, Vol. 24, No. 6, 2010, pp. 945-946. doi:10.1089/end.2009.0505

[15] A. C. Mues, J. A. Graversen, M. D. Truesdale, C. Casazza and J. Landman, "BioGlue Iceball Stabilization to Mini- mize the Risk of Hemorrhage during Laparoscopic Renal Cryoablation," Urology, Vol. 78, No. 2, 2011, pp. 353356. doi:10.1016/i.urology.2011.01.072

[16] K. S. Hafez, A. C. Novick and S. C. Campbell, "Patterns of Tumor Recurrence and Guidelines for Followup after Nephron Sparing Surgery for Sporadic Renal Cell Carcinoma," The Journal of Urology, Vol. 157, No. 6, 1997, pp. 2067-2070. doi:10.1016/S0022-5347(01)64675-5

[17] P. H. Van, B. Bamelis, R. Oyen and L. Baert, "Partial Nephrectomy for Renal Cell Carcinoma Can Achieve Long-Term Tumor Control," The Journal of Urology, Vol. 160, No. 3, 1998, pp. 674-678.

[18] R. Zigeuner, F. Quehenberger, K. Pummer, P. Petritsch and G. Hubmer, "Long-Term Results of Nephron-Sparing Surgery for Renal Cell Carcinoma in 114 Patients: Risk Factors for Progressive Disease," BJU International, Vol. 92, No. 6, 2003, pp. 567-571. doi:10.1046/j.1464-410X.2003.04414.x

[19] K. S. Hafez, A. C. Novick and S. C. Campbell, "Patterns of Tumor Recurrence and Guidelines for Followup after Nephron Sparing Surgery for Sporadic Renal Cell Carcinoma," The Journal of Urology, Vol. 157, No. 6, 1997, pp. 2067-70. doi:10.1016/S0022-5347(01)64675-5

[20] M. S. Lee, T. O. Young, K. H. Woong, H. R. Koon, D. C. Young, J. H. Sung, C. Y. Seung and W. K. Ki, "CT Findings after Nephron-Sparing Surgery of Renal Tumors," American Journal of Roentgenology, Vol. 189, No. 5, 2007, pp. 264-271. doi:10.2214/AJR.07.2542

[21] D. Pai, J. M. Willatt, M. Korobkin, R. H. Cohan, J. H. Ellis, I. R. Francis, J. S. Wolf and M. Schipper, "CT Appearances Following Laparoscopic Partial Nephrectomy for Renal Cell Carcinoma Using a Rolled Cellulose Bolster," Cancer, Vol. 10, No. 1, 2010, pp. 161-168. doi:10.1102/1470-7330.2010.0023 